\title{
Neutron Spectra Unfolding with Minimum Fisher Regularisation
}

\author{
J. Mlynar ${ }^{* 1}$, L. Bertalot ${ }^{2}$, M. Tsalas $^{3}$, G. Bonheure ${ }^{4}$, S. Conroy ${ }^{5}$ and JET-EFDA \\ contributors $^{6}$ \\ EFDA JET, Culham Science Centre, OX11 3DB Abingdon, United Kingdom \\ ${ }^{1}$ Association EURATOM-IPP.CR, Za Slovankou 3, 18221 Prague 8, Czech Republic \\ ${ }^{2}$ Association EURATOM-ENEA sulla Fusione, Frascati, C.P. 65, 00044-Frascati, Italy \\ ${ }^{3}$ Association EURATOM-Hellenic Republic, N.C.S.R. "Demokritos", 15310 Athens, Greece \\ ${ }^{4}$ Association EURATOM-Etat Belge, LPP-ERM/KMS, B-1000 Brussels, Belgium \\ ${ }^{5}$ Association EURATOM-VR, Uppsala University, SE-751 05 Uppsala, Sweden \\ ${ }^{6}$ See annex of J. Pamela et al, “Overview of JET Results “, Nucl. Fus. 45 (2005) S63 \\ E-mail: jan.mlynarejet.efda.org
}

During past experimental campaigns at JET, the NE213 liquid scintillator proved to be a valid diagnostic tool for spectral measurements of fusion neutrons emitted from the plasma. The scintillator response function to neutrons is derived from theoretical estimation combined with measurements at an accelerator-based neutron source. The spectrum unfolding poses an illconditioned inversion problem similar to tomographic reconstruction.

Minimum Fisher Regularisation (MFR) is one of the established methods in plasma 2D tomography, providing a rapid and robust tool for sparse data inversion without special a priori assumptions. In neutron analyses at JET, the MFR has been applied both in tomography (see contribution by G. Bonheure) and in unfolding the NE213 spectra, where good agreement with results of the standard Maximum Entropy unfolding procedure (MAXED) was demonstrated.

This contribution is focussed on further progress in the MFR application to the unfolding of NE213 spectra. In particular, the L-curve maximum curvature optimisation was implemented which gives MFR the full independence required for automated operation. New results are presented in comparison with the MAXED analyses. The differences between the data and the retrofit of the unfolded spectra are studied and the MFR potential for future applications is outlined.

International Workshop on Fast Neutron Detectors

University of Cape Town, South Africa

April 3-6, 2006

\section{${ }^{*}$ Speaker}




\section{Introduction}

In this contribution the Minimum Fisher Regularisation (MFR) spectra unfolding method is presented in the framework of analyses of data from the $\varnothing 50 \times 20 \mathrm{~mm}$ NE213 scintillation detector installed in the JET roof laboratory [1]. JET (Joint European Torus, [2]) is the world biggest tokamak and currently the only fusion facility able to operate with tritium. Therefore, the facility plays a key role in development of fusion-relevant neutron diagnostics [3].

NE213 scintillation detectors are widely used for fast neutron measurements due to their simplicity, good neutron-gamma discrimination and high light output. However, the neutron energy spectrum $\Phi\left(E_{n}\right)$ has to be unfolded from the light pulse height spectrum $L\left(E_{l}\right)$ registered by a photomultiplier as a result of neutron-proton recoil in NE213 organic liquid scintillator. A semi-empirical relation can be introduced

$$
L_{i}=\sum_{j}^{N} R_{i j} \Phi_{j}+\tilde{\varepsilon}_{i}
$$

where the constant response matrix $R_{i j}$ of the detector is given by theoretical predictions combined with accelerator calibrations [4], $\mathrm{N}$ is the number of neutron energy bins and the corrections $\widetilde{\varepsilon}_{i}$ reflect existing statistical and systematic errors. The set of eqs. (1) need to be inverted in order to obtain the neutron spectrum from the measured pulse-height spectrum. The task of determining the unknown $\Phi_{j}$ from the observable $L_{i}$ presents an ill-conditioned problem, with high risk of producing large artefacts due to errors, even if the values of $\widetilde{\varepsilon}_{i}$ are low. At JET, the maximum entropy deconvolution code MAXED [5] has been used for the NE213 data unfolding, and the MFR was introduced in order to confirm these results independently [6].

Originally, MFR has been applied in plasma tomography [7] where the relation between the measured line integrals and unknown local emissivities is reminiscent of Eq. (1). The MFR method proved to provide rapid and robust solutions to this algebraic problem, and due to the similarity between spatial and spectral inversions, the modification of the MFR algorithm to NE213 unfolding code [6] was straightforward.

\section{Minimum Fisher Regularisation with the L-curve principle}

MFR belongs to the class of Tikhonov's methods [8]. In the following, the principles are summarised. For a detailed description see [7]. Solutions to the set of eq. (1) is found in the form of a constant reconstruction matrix $M_{j i}$ :

$$
\Phi_{j}=\sum_{i}^{T} M_{j i} L_{i}
$$


where $T$ is the number of pulse-height energy bins. The set of eq. (2) is obtained from (1) using pre-defined constraints on the unknowns $\Phi_{j}$ (typically, some sort of smoothness is required), while keeping the expected data errors $\sigma_{i}$ - i.e. data errorbars, see eq. (5) - close to residuals $\varepsilon_{i}$. The residuals are defined by a retrofit given by substition of eq. (2) into eq. (1). Notice that the residuals $\varepsilon_{i}$ are not identical to the (unknown) corrections $\widetilde{\varepsilon}_{i}$ as eq. (2) provides only a fitted solution.

In the MFR method a smoothness constraint on $\Phi_{j}$ is introduced in a form of a discrete iteration towards the Minimum Fisher Information principle, where the Fisher information is defined by

$$
I_{F}=\int \frac{1}{\Phi\left(E_{n}\right)}\left(\frac{d \Phi\left(E_{n}\right)}{d E_{n}}\right)^{2} d E_{n}
$$

with $E_{n}$ the neutron energies. The algorithm finds - among the infinite number of possible solutions that would fit within the data errorbars - a smooth solution with the lowest amount of Fisher information, thus effectively detailing regions with higher neutron intensities. The minimum Fisher information approach seems particularly suitable for analysis of pulse-height spectra.

Reliable performance of MFR is conditioned by a good estimate of the uncertainties - i.e., of the individiual expected errors $\sigma_{i}$. The next challenge of key importance is to quantify the "close" relation between the expected errors and the resulting residuals, which corresponds to the overall smoothness of the unfolded spectrum. A unique dedicated regularisation parameter $\lambda$ controls the relation in MFR. So far, the $\lambda$ value has been looked for in an iterative $\chi^{2}$-like test:

$$
\chi^{2}=\frac{1}{T} \sum_{i}^{T}\left(\frac{\varepsilon_{i}}{\sigma_{i}}\right)^{2}
$$

where the $\chi^{2}$ iteration should in principle approach unity. However, the condition $\chi^{2}=1$ is not completely correct for many problems of data analysis for several reasons. In particular, the estimate of the uncertainties $\sigma_{i}$ need not be valid, for example see end of part 3. There is also a quite loose link between the ideal corrections $\widetilde{\varepsilon}_{i}$ and the residuals $\varepsilon_{i}$. As a consequence, in MFR the condition results in ocassional oversmoothing or overfitting, so that manual "tuning" of the $\chi^{2}$ target - based on visual validation of the result - is often required [6].

In order to amend this, the L-curve principle [8] has been implemented for the first time in MFR. The L-curve is a logarithmic plot of the norm of the constraints versus the norm of the residuals. In case of MFR, the two respective norms $\|H \Phi\|$ and $\|R \Phi-L\|$ are given by

$$
\|H \Phi\|=\sqrt{\sum_{i}^{N} \sum_{j}^{N} \Phi_{i} H_{i j} \Phi_{j}} \quad\|R \Phi-L\|=\sqrt{\chi^{2}}
$$


where $H$ is the smoothing matrix, see eqs. (18) and (25) in [7]. According to the L-curve principle it is argued in [8] that the "corner" of the $\|H \Phi\|$ versus $\|R \Phi-L\|$ plot (on the log-log scale) gives a near-optimal regularisation parameter. Figure 1 shows the L-curve and its "corner" as obtained for real NE213 data (corresponding to the spectrum in Fig. 3).

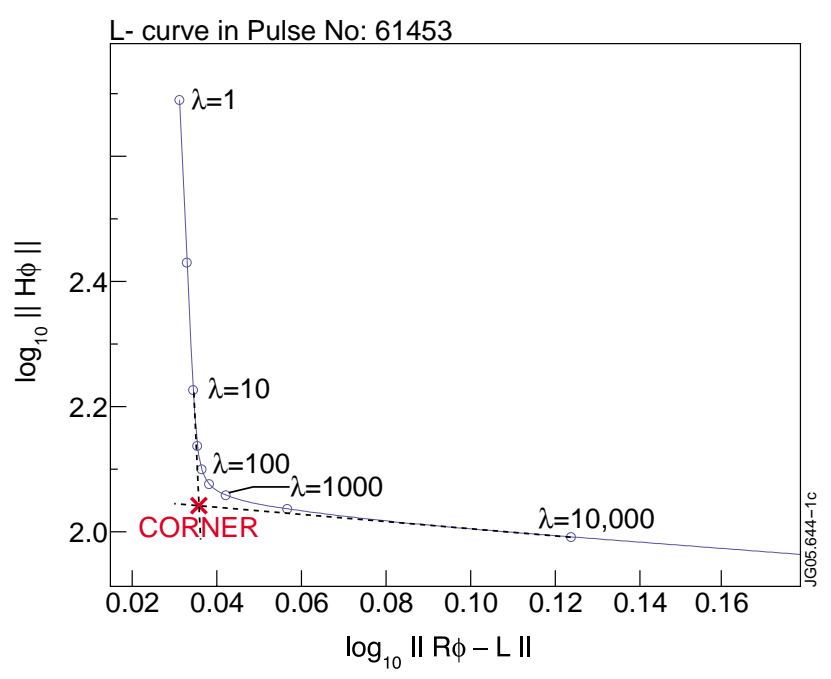

Figure 1: The L-curve principle corresponding to Fig.3

\section{Recent results}

Spectra presented in this contribution correspond to data from the $\varnothing 50 \times 20 \mathrm{~mm}$ NE213 scintillator detector obtained during the JET Trace Tritium Experiments in 2003, when the rates of D-D and D-T neutrons were comparable [2]. The choice of experimental discharges complements previous results presented in [6]. The analyses also served to validate the L-curve functionality in MFR, that is, no additional constraints or parameters were input to the algorithm except the choice of the range of applied data bins.

Figure $2 \mathrm{a}$ shows the unfolded neutron spectrum corresponding to D-D neutrons (deuterium-deuterium fusion neutrons, $2.45 \mathrm{MeV}$ in the rest frame) integrated over six seconds in the JET discharge No. 62752. In this experiment, low density low temperature plasma ( $n \sim$ $510^{19} \mathrm{~m}^{-3}, T_{i} \sim 2-3 \mathrm{keV}$ ) was heated by $3 \mathrm{MW}$ Deuterium Neutral Beam Injection only. Most of the D-D fusion reactions are due to beam-plasma ion collisions, which may explain the neutron spectrum peak splitting. The beam particles, ionised by the plasma and captured by the JET magnetic field, rotate at high velocities perpendicular to the field lines, which influences the energies of the D-D neutrons (see also [9]). The minor peak at $1.9 \mathrm{MeV}$ is supposedly due to neutron in-scatter in the detector's collimator. Notice that the two maxima have approximately the same height when the in-scatter is deducted. As expected, MAXED provides qualitatively the same result as MFR, with value of $\chi^{2}$ found by the L-curve principle slightly lower than unity (see Fig. 2a). 


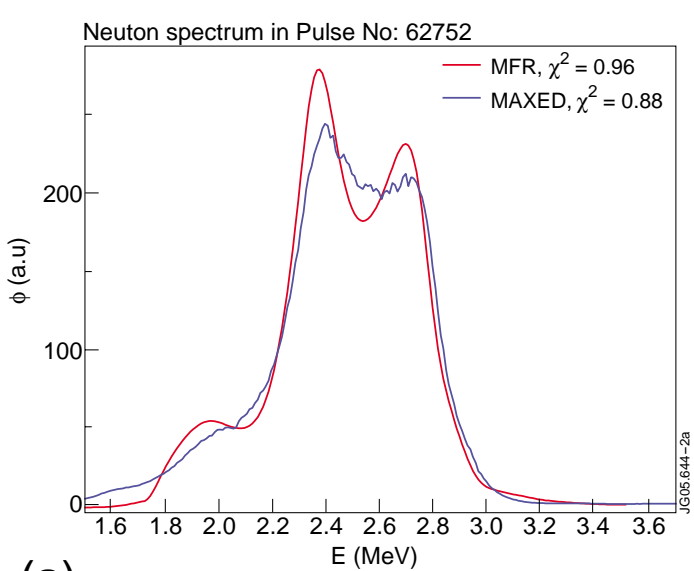

(a)

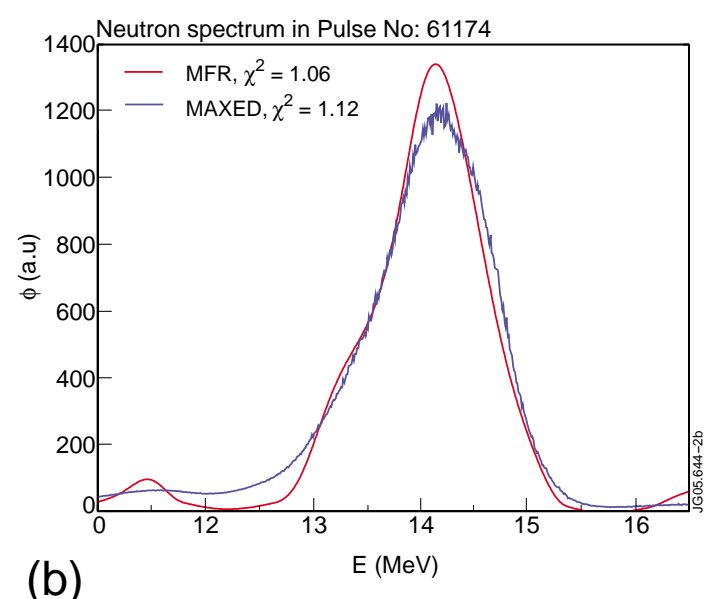

(b)

Figure 2: Two examples of the NE213 neutron spectra in plasmas with the neutral beam heating:

(a) D-D fusion spectrum (b) T-D fusion spectrum

To compare, figure $2 \mathrm{~b}$ presents the unfolded T-D neutron spectrum (spectrum of tritiumdeuterium fusion neutrons, $14 \mathrm{MeV}$ in the rest frame) registered by the NE213 detector in the JET discharge No. 61174. This medium-density discharge $\left(n \sim 1.510^{20} \mathrm{~m}^{-3}\right)$ had tritium puff and 13 MW Deuterium Neutral Beam Injection (NBI). As in the D-D case, the fast beam particles are likely to be responsible for the neutron peak distortion. The shape of the peak reflects more complicated trajectories of the fast particles, as demonstrated already in [9]. There is reasonable agreement between the MFR and MAXED reconstructions, namely in terms of peak width and asymmetry, with value of $\chi^{2}$ slightly above one. However, MFR does not show any in-scatter.

Figure 3 details results obtained in the unfolding of another T-D neutron spectrum, corresponding to the JET discharge No. 61451. This experiment was similar in density and tritium fuelling to the one in Fig. 2b, but in this case plasma was heated only by $1.7 \mathrm{MW}$ Ion Cyclotron Resonant Heating with frequency tuned on tritium minority ions [10]. Figure 3a shows data (the pulse height spectrum in electron equivalent energy units) and the energy interval delimited for the unfolding process, which totals 228 energy bins. Figure $3 \mathrm{~b}$ shows the resulting neutron spectrum (with 910 energy bins) compared with the MAXED reconstruction. A deeper understanding of the shape of the observed neutron spectrum would require an investigation of the underlying RF acceleration mechanisms involved. Similarly to Fig. $2 b$, the MFR reconstruction does not indicate any important neutron in-scatter. Both results are retrofitted using Eq. (1) to Fig. 3a. Fig 3c shows residuals normalised to the errorbars $\left(\chi_{i}=\varepsilon_{i} / \sigma_{i}\right)$, the difference between MAXED and MFR retrofits, and the $\chi_{i}$ value averaged over 7 neighbour bins. These averaged residuals show collective and rather periodic behaviour on the scale of hundreds of e-keV, confirming similar observations in [6]. This is likely to be due to systematic errors either in the reconstruction matrix, or in performance of the diagnostics hardware, and demonstrates the importance of a precise detector calibration. 

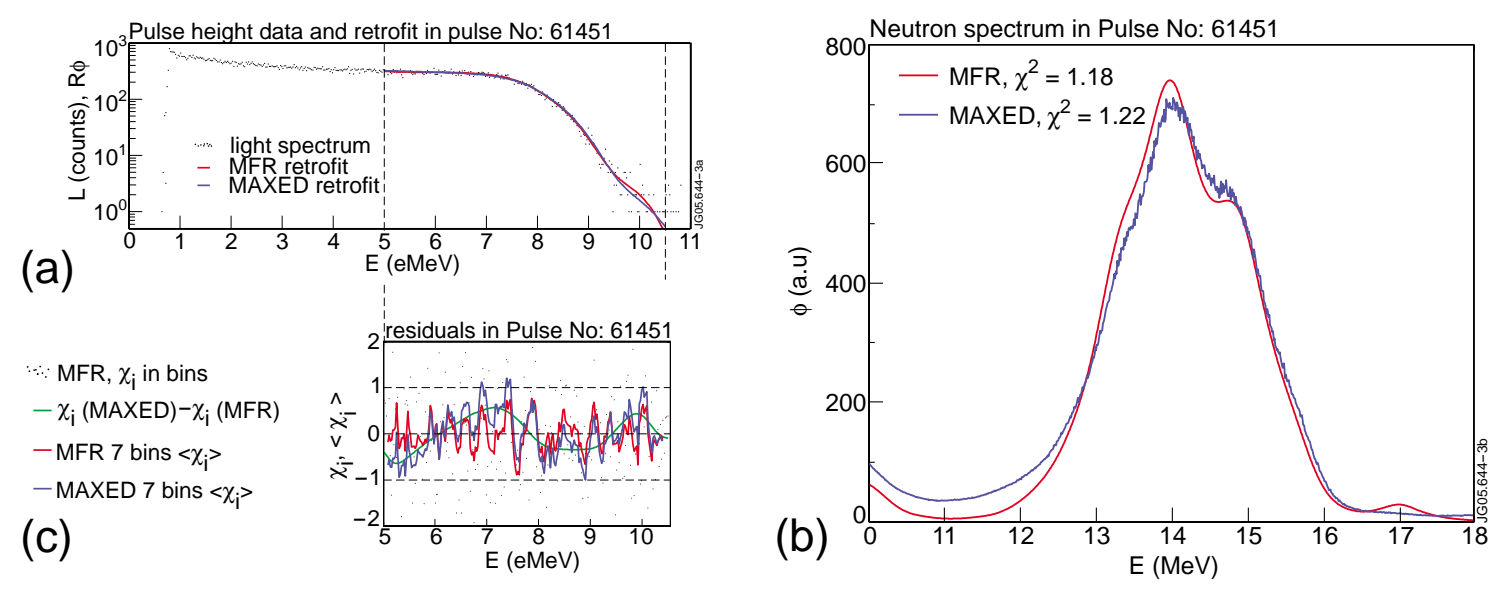

Figure 3: NE213 neutron spectrum in a JET plasma with Ion Cyclotron Resonant Heating:

(a) pulse height spectrum showing the retrofit, (b) unfolded spectrum of neutrons, (c) residuals $\varepsilon_{i} / \sigma_{i}$

The difference between MAXED and MFR retrofits in Fig. 3c deserves further discussion ${ }^{*}$. Importantly, the difference stays within data errorbars so that it can be concluded that both methods provide an acceptable solution to the unfolding problem. It is however obvious that the MFR fit is locally closer to the data, while MAXED result in a solution with minor systematic deviations from the average. For example, the observed deviation between 5.5 to $7 \mathrm{eMeV}$ accounts for the higher levels of in-scatter in MAXED as compared to MFR. This behaviour is likely to be due to MAXED global constraints. Discussion and validation of these constraints is beyond the scope of this paper.

MFR is based rather on local (smoothing) constraints so that its performance critically depends on a correct description of the data errors. In the present version it has been supposed that the expected errors in the NE213 data are equal to the square root of the bin counts. However, it has been noticed that the MFR reconstructed T-D spectra tend to overfit at low neutron energies and, indeed, data statistics in Fig. 3a plateau indicate a statistical error above the square root of counts, approximately corresponding to an additional 3\% data uncertainty. When this is accounted for in the form of percentual "white noise" in the MFR algorithm, then the L-curve finds value of $\chi^{2}$ equal to the expected unity. Unfortunately the impact on the shape of the neutron spectrum is negligible. Although the issue needs further investigation, it seems that the MFR fit is either correct or misled by systematic errors in data and/or the response matrix.

\section{Conclusions}

Neutron spectra unfolded by the upgraded Minimum Fisher Regularisation method are in good agreement - within the data errorbars - with the MAXED results, which confirms previous

\footnotetext{
* Notice that the retrofit procedure is a straightforward response matrix multiplication and as such it is fully independent of the unfolding method.
} 
results published in [6]. Furthermore, the present analyses strongly indicate that the L-curve "corner" indeed provides a suitable value for the regularisation parameter, which makes MFR fully independent of free parameters. Therefore, with the L-curve upgrade and due to relatively high algorithm speed, MFR becomes a suitable candidate for intensive computation tasks like iterative cross-checks of response matrix consistency in the detector calibration, automated post-discharge spectra unfolding, or even approximate real-time spectra unfolding that may be important for the future reactor-grade fusion facilities including ITER.

In the near future, the new fast digital data acquisition system for the nineteen NE213 detectors in the neutron profile monitor [3] can widen the scope for the unfolding of neutron spectra using the MFR method, provided that the response matrices are carefully determined.

It can be summarized that the quality of response matrix - i.e. precise detector calibration, its reliable collimation and spectral stability in time - are of key importance for dedicated neutral spectral measurements using NE213 detectors. Systematic errors considerably reduce resolution and reliability of any unfolding method, and they are difficult to track down. Detailed calibrations as well as potentially higher count rates with the new digital data acquisition are planned for the NE213 detector in the near future. Improved data statistics due to a higher count rate would further enhance reliability of the unfolding process, however, this will be in competition with the possibility of increased time resolution of the neutron spectra measurements.

\section{References}

[1] A. Zimbal et al., High Resolution Neutron Spectrometry with Liquid Scintillation Detectors for Fusion Plasmas, in proceedings of this workshop, OE06

[2] J. Pamela, J. Ongena and JET EFDA Contributors, Overview of JET results, Nuclear Fusion 45 (2005) S63-S85

[3] G. Bonheure et al., Neutron Diagnostics for Reactor Scale Fusion Experiments: A Review of JET Systems, in proceedings of this workshop, RE02

[4] N.P. Hawkes et al., Measurements of the proton light output function of the organic liquid scintillator NE213 in several detectors, Nucl. Instr. and Meth. 476 (2002) 190-194

[5] M. Reginatto, P. Goldhagen, S. Neumann, Spectrum unfolding, sensitivity analysis and propagation of uncertainties with the maximum entropy deconvolution code MAXED, Nucl. Instrum. Methods A476 (2002) 242-786

[6] J. Mlynar, J.M. Adams, L. Bertalot, S. Conroy, First results of Minimum Fisher Regularisation as unfolding method for JET NE213 liquid scintillator neutron spectrometry, Fusion Engineering and Design 74 (2005) 781-786

[7] M. Anton et al., X-ray tomography on the TCV tokamak, Plasma Phys. Control. Fusion 38 (1996) 1849-1878

[8] P. Ch. Hansen, Numerical tools for analysis and solution of Fredholm integral equations of the first kind, Inverse Problems 8 (1992) 849-872

[9] M.J. Loughlin et al., Consequences of trapped beam ions on the analysis of neutron emission data, in proceedings of 16th EPS Conference on Controlled Fusion and Plasma Physics, Venice 1989, Ed. S.Segre, H. Knoepfel, E.Sindoni, 83-85

[10] P. U. Lamalle et al., Expanding the operating space of ICRF on JET with a view to ITER, Nucl. Fus. 46 (2006) 391-400 